\title{
sciendo
}

\section{MBboard: Validity and Reliability of a New Tool Developed to Evaluate Specific Strength in Rock Climbers}

\author{
by \\ Thomas K. Marino ${ }^{1}$, Daniel B. Coelho ${ }^{2}$, Adriano E. Lima-Silva3 , Romulo Bertuzzi ${ }^{1}$
}

\begin{abstract}
In the present study, we analysed the validity and reliability of a new tool designed to assist the measurement of maximal upper-limb strength in rock climbers in a specific way, named MBboard. The MBboard consists of an artificial small climbing hold affixed to a wooden board, which is connected to any cable-motion strength equipment to determine the maximum dynamic strength (MBboard-1RM). Ten male rock climbers (Rock Climbing Group, $R C G=$ 10 ) and ten physically active men (Control Group, $C G=10$ ) performed, on three separate occasions, a familiarization session with procedures adopted during MBboard-1RM testing and two experimental trials (i.e., test and retest) to determine the construct validity and reliability of the MBboard during unilateral seated cable row exercise. In the first trial, the electromyographic activity (EMG) was recorded from the flexor digitorum superficialis. The self-reported climbing ability was also recorded. The RCG had superior performance (i.e. $37.5 \%$ ) and EMG activity (i.e. 51\%) in MBboard-1RM testing when compared with the CG $(p<0.05)$. There was a significant correlation between the MBboard-1RM results and climbing ability $(r>0.72, p<0.05)$. Intraclass correlation coefficient analysis revealed good reliability within trials (ICC $>0.79, p<0.05$ ). These findings suggest that the MBboard is a valid and reliable tool to assess rock climbing-specific maximal strength. The validity of MBboard-1RM appears to be related to the finger flexor muscles activation, probably reflecting the specific adaptations resulting from long-term practice of this sport discipline.
\end{abstract}

Key words: rock climbing, physical evaluation, maximum dynamic strength, electromyographic activity.

\section{Introduction}

Indoor sport climbing has experienced an increase in its popularity in both recreational and competitive terms, with its first participation in the Olympic Games in Tokyo 2021. This has led some researchers to focus on the analysis of the possible relationships between climbing performance and anthropometric (Grant et al., 2001; Watts et al., 1993), biomechanical (Bourdin et al., 1998; Quaine et al., 1997) and physiological (Bertuzzi et al., 2007; Magiera et al., 2018; Mermier et al., 2000) variables. Previous findings suggest that maximal upper limb strength is a key factor for successful climbing performance
(Mermier et al., 2000), with particular relevance for finger flexors (Magiera et al., 2013; Michailov et al., 2018). It has been demonstrated that welltrained climbers possessed a higher handgrip force (Quaine et al., 2003) and a higher activation of the flexor digitorum superficialis (FD) when compared with non-climbers during a pull-up climbing movement (Koukoubis et al., 1995). These neuromuscular adaptations have been attributed to high-intensity isometric forearm muscle contractions often performed by climbers during ascents of climbing routes (Baláš et al., 2015; Michailov et al., 2014).

\footnotetext{
1 - Endurance Performance Research Group (GEDAE-USP), School of Physical Education and Sport, University of São Paulo, São Paulo, SP, Brazil.

2 - Center of Engineering, Modelling and Applied Social Science, Federal University of ABC, São Paulo, Brazil.

3 - Human Performance Research Group, Academic Department of Physical Education (DAEFI), Technological Federal University of Parana, Curitiba, PR, Brazil.
} 
The relevance of handgrip force for sport climbing performance has been established through evaluation based on traditional and adapted handgrip dynamometry (Quaine et al., 2003; Thompson et al., 2015), which involves a certain financial cost and the need for specialized evaluators. Those characteristics of evaluation may restrict the number of climbers who may have access to the measurement of specific maximal handgrip force, impacting the development of sports training. It is also important to note that, while forearm muscles are required to grip the artificial holds, other muscles of the upper-limbs (i.e., posterior deltoid) are dynamically recruited in order to balance and displace the entire body on inclined walls (Vigouroux et al., 2015). As a possible result, the measurement of strength using traditional or adapted dynamometers is limited by analysing only the forearm muscles, disregarding the importance of other upper limb muscles for climbing performance. Considering these aspects, it would be important to develop new low-cost tools able to assist the measurement of maximal strength of the upper limbs of climbers in a more specific manner.

Therefore, the aim of the present study was to develop a low-cost and easy-to-build tool for measurement of the specific maximal upperlimb strength in rock climbers, named the MBboard. We also described evidence of validity (i.e., construct validity) and reliability (i.e., test and retest analysis) of the MBboard. It was hypothesized that: i) maximum dynamic strength measured by the MBboard (MBboard-1RM) would be able to discriminate between individuals with different levels of rock climbing experience, mainly due to a higher activation of the FD; ii) the performance measured on the MBboard-1RM would be correlated with climbing ability; and iii) the MBboard-1RM would be reliable when analysed in test and retest trials.

\section{Methods}

\section{Participants}

Ten male rock climbers [rock climbing group $(\mathrm{RCG})=10$; age $=28 \pm 6$ years, body mass $63.7 \pm 3.5 \mathrm{~kg}$, body height $175.2 \pm 5.1 \mathrm{~cm}$ ] and ten physically active men [control group $(\mathrm{CG})=10$, age $=23 \pm 3$ years, body mass $72.5 \pm 6.3 \mathrm{~kg}$, body height $178.1 \pm 6.3 \mathrm{~cm}$ ] voluntarily participated in the study. The RCG consisted of subjects who were practicing indoor rock climbing for at least 1 year and could climb difficult routes. Selfreported climbing ability was expressed by their higher redpoint grade climbed over the last year and converted to the International Rock Climbing Research Association Scale (Draper et al., 2016) (17 \pm 3 IRCRA Scale), similar to a recent study (Michailov et al., 2018). The CG consisted of subjects who were engaged in recreational sports (i.e., jogging, soccer, basketball and cycling) 3-4 times per week for at least one year. As exclusion criteria, participants should not present any cardiorespiratory disease, not be engaged in any medical treatment, not use anabolic steroids, and no suffer from any recent injuries that could compromise their participation. Participants were informed about the risks associated with the study protocol and signed a consent form agreeing to participate in the experiments. This investigation was approved by the Ethics Committee of the University of Sao Paulo for Human Studies (process 2.942.117), in accordance with the Declaration of Helsinki.

\section{Design and Procedures}

Experimental design

Participants visited the laboratory on three separate occasions over a 2-wk period, at least 48 hours apart. All tests were performed at the same time of day to avoid the effects of circadian variation. During the first session, participants underwent anthropometric measurements (i.e., body mass and height) and were familiarized with all MBboard-1RM procedures. The MBboard-1RM was performed during the second (i.e., test) and third (i.e., retest) session. During the experimental sessions, maximum strength of the upper limbs and electromyographic activity (EMG) were recorded. Participants were instructed to refrain from exhaustive or unaccustomed exercise, alcohol and caffeine 48-h before the experimental sessions.

Technical characteristics of the MBboard

Figure 1 shows a schematic representation of main characteristics of the MBboard. The MBboard is composed of an artificial small climbing hold (with approximately $2.5 \mathrm{~cm}$ rounded edge depth) 
affixed to a wooden board ( $30 \mathrm{~cm}$ length by 17.5 $\mathrm{cm}$ width). There is a hole in the wooden board in which a carabiner is placed to connect the MBboard to the crossover machine (or cablemotion strength equipment). On the bottom of the wooden board, there are two parallel holes which serve to place a small cord that surrounds the forearm for the stabilization of the MBboard during exercise.

Maximum dynamic strength measured by the MBboard

Participants were familiarized with all the procedures, equipment, and proper exercise techniques prior to data collection. An adjustable-height bench press seat was used to keep the knees $\left(90^{\circ}\right)$, trunk $\left(90^{\circ}\right)$, and arms $\left(90^{\circ}\right)$ flexion angles constant during each MBboard1RM repetition. At the beginning of each set, participants were instructed to grip the climbing hold and start the MBboard-1RM with the elbow extended and finish it with the elbow flexed at approximately $90^{\circ}$ during the unilateral seated cable row exercise (Figure 2). The participants' settings on the crossover machine were recorded to guarantee the same positioning across familiarization and experimental sessions. Participants were free to choose the grip technique during familiarization, which was recorded and replicated in the experimental sessions.

The MBboard-1RM was evaluated on the dominant and non-dominant upper-limb, which was defined based on the subject daily activities (Coley et al., 2008), according to standard procedures for maximum dynamic strength measurement (Brown and Weir, 2001). Participants performed a brief general warm-up composed of a 5 -min run at $8 \mathrm{~km} \cdot \mathrm{h}^{-1}$ followed by light upper limb stretching. Following the initial warm-up run, participants performed a specific warm-up composed of three sets, interspaced by a 3-min rest interval. In the first set, five repetitions with the load of $5 \mathrm{~kg}$ were performed. In the second set, five repetitions at $50 \%$ of the estimated MBboard-1RM were completed. The third set of the warm-up was composed of three repetitions at $70 \%$ of the estimated MBboard1RM. After the third set, participants rested for three minutes before performing five trials to achieve the MBboard-1RM, which was defined as the maximum weight that could be lifted once using proper technique. A three-minute rest interval of passive recovery was allowed between the attempts. Participants were allowed to use chalk as a natural practice in climbing and the hold was cleaned with a brush when needed. Electromyographic activity (EMG)

During the specific warm-up protocol (i.e., load of $5 \mathrm{~kg}$ ) and MBboard-1RM measurement, EMG signals were recorded from the right arm. Disposable dual $\mathrm{Ag} / \mathrm{AgCl}$ snap electrodes with a 1-cm diameter and 2-cm centerto-center spacing (Noraxon, Scottsdale, AZ, USA) were placed on the belly of the flexor digitorum superficialis (FD) and posterior deltoid (PD) muscle before starting the test. The SENIAM guidelines were followed for skin preparation, electrode placement, and orientation. Electromyographic signals were registered with a telemetric EMG system, which had a gain of $1000 \mathrm{~Hz}$, a bandwidth $(-3 \mathrm{~dB})$ of 10 to $500 \mathrm{~Hz}$, and a common mode rejection ratio $>85 \mathrm{~dB}$. The signal was relayed to the computer via a 16-bit A/D converter (Myotrace 400, Noraxon, Scottsdale, AZ, USA). Only the concentric phase was included, and only the iEMG signal of the maximal repetition was chosen for subsequent statistical analyses. The period of activation was determined as the period where the signal was above a threshold of $15 \%$ of the maximum activity of that muscle during the test for at least $100 \mathrm{~ms}$ (Damasceno et al., 2014, 2015). The root mean square (RMS) of the EMG signal recorded during the MBboard-1RM test was normalized to the RMS recorded during the last muscle contraction measured during the specific warmup protocol (i.e. load of $5 \mathrm{~kg}$ ).

\section{Statistical analysis}

Data distribution was analysed by the Shapiro-Wilk test and the results showed a normal Gaussian distribution. Data are reported as mean and standard deviation (SD). RCG and CG data were compared by an unpaired $t$-test. The Pearson product-moment coefficient was used to determine the relationship between MBboard-1RM and climbing experience for the RCG. Intraclass correlation coefficient (ICC) was used to determine the reliability of the MBboard$1 \mathrm{RM}$ for the RCG during test and retest sessions, assuming an ICC value $<040$ as poor, between 0.40 and 0.70 as fair, between 0.70 and 0.90 as good and $>0.90$ as excellent (Coppieters et al., 
2002). Statistical significance was set at $p<0.05$. All analyses were performed using the SPSS program (version 13.0, Chicago, USA).

\section{Results}

Validity and reliability of MBboard-1RM

Figure 3 shows the maximum dynamic strength measured by the MBboard (i.e., test and retest MBboard-1RM) for both the CG and the RCG, as well as the correlation between MBboard-1RM and climbing ability self-reported by the RCG. The RCG had a superior performance in MBboard-1RM when compared with the CG for both dominant ( $p<0.01$, Panel A) and non-dominant $(p<0.01$, Panel B) upperlimbs. There were significant Pearson productmoment correlations between MBboard-1RM performed on dominant $(\mathrm{r}=0.73, p=0.02$, Panel
C) and non-dominant $(r=0.72, p=0.02$, Panel D) upper-limbs with climbing ability. ICC analysis revealed good reliability between the trials for both dominant (ICC $=0.79, p=0.01$ ) and nondominant $($ ICC $=0.85, p<0.01)$ upper-limbs in RCG.

Surface electromyographic activity during MBboard1RM

Figure 4 shows EMG activity for the flexor digitorum superficialis (panel A) and posterior deltoid (panel B) during MBboard-1RM evaluation. EMG activity for the flexor digitorum superficialis was significantly higher for the RCG $(p<0.01)$ when compared with the CG, while there were non-significant differences between the RCG and the CG $(p=0.11)$ in EMG activity for the posterior deltoid.

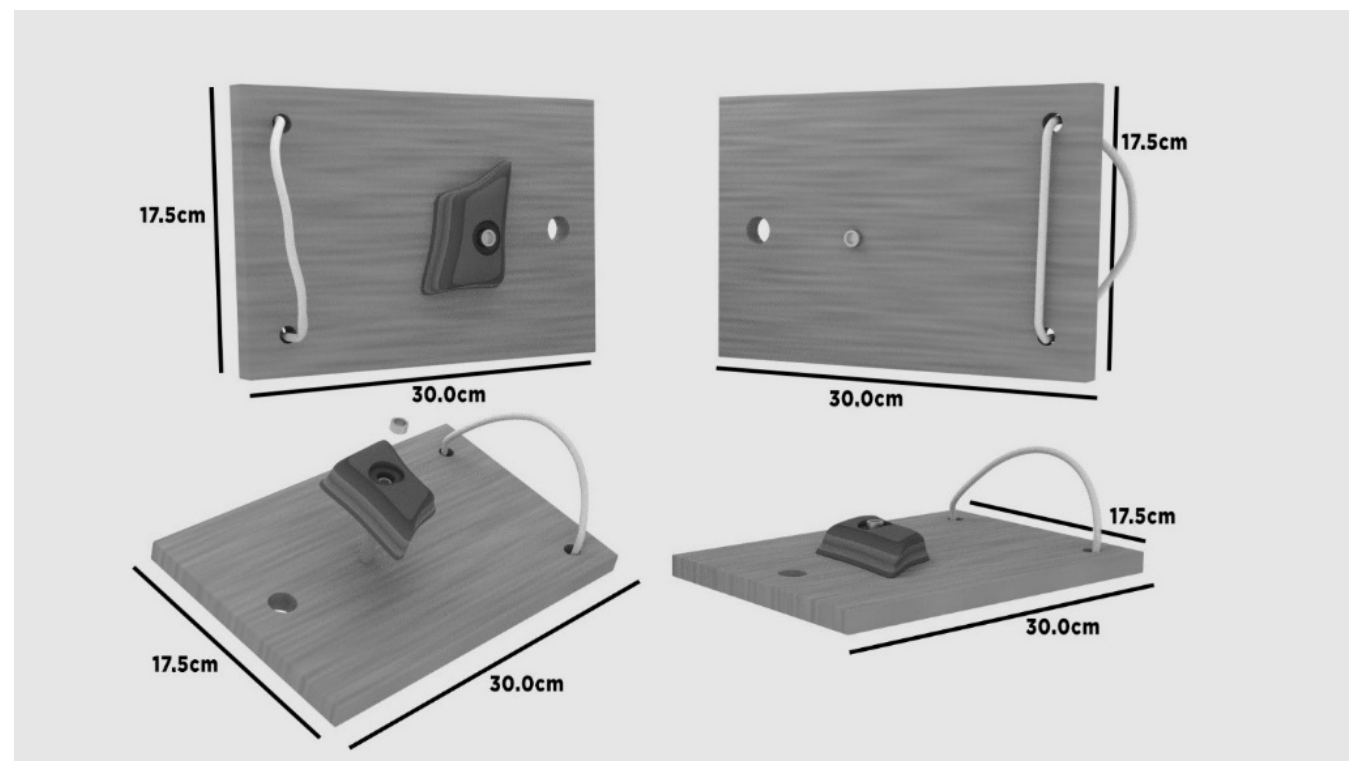

Figure 1

Schematic representation of main characteristics of the Mbboard. 

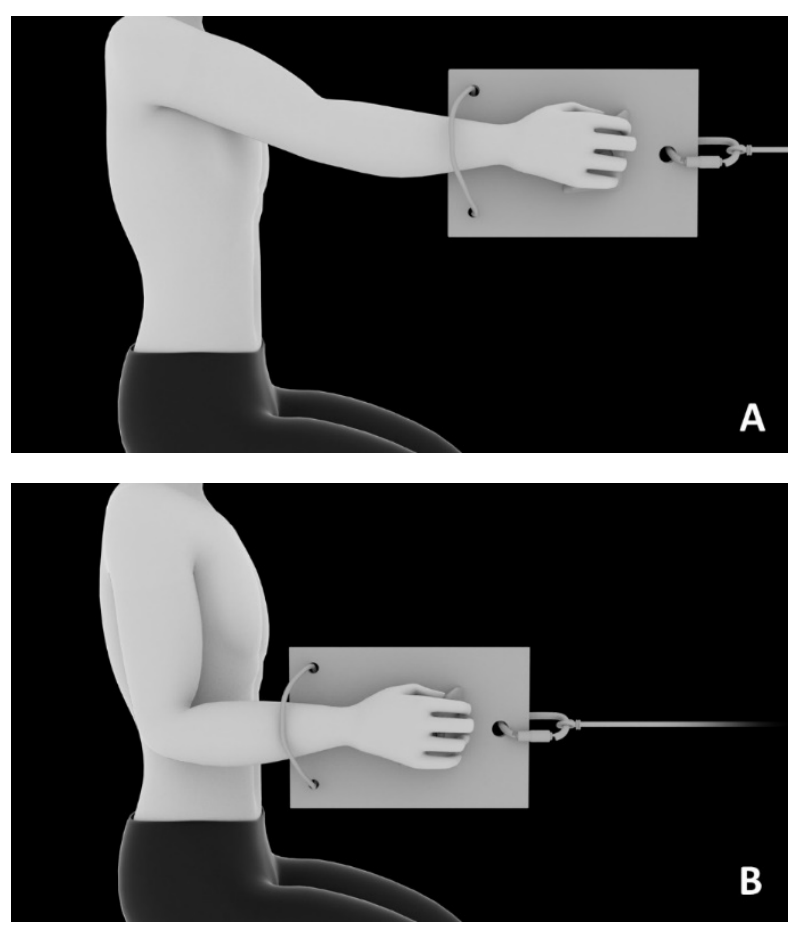

Figure 2

Schematic representation of initial (panel A) and final (panel B) arm positions during MBboard-1RMassessment.

A

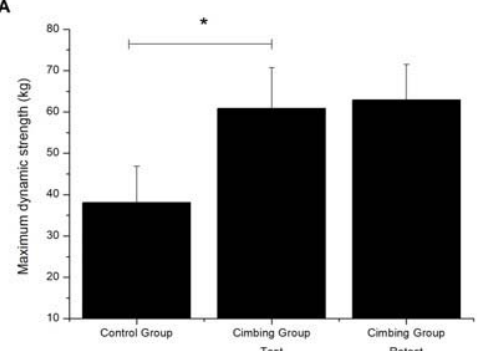

c

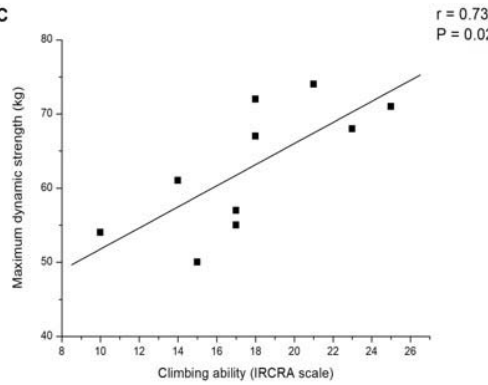

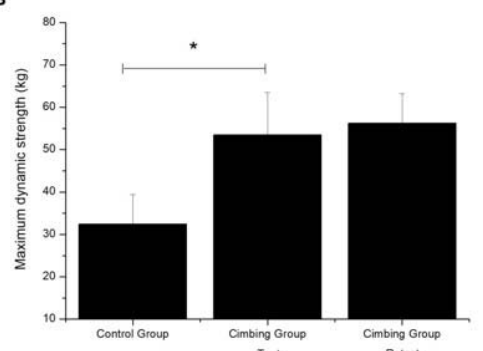

D

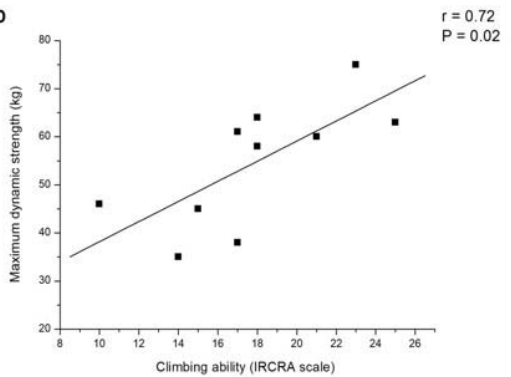

Figure 3

Maximum dynamic strength measured using the MBboard (Panel A: dominant upper-limb, Panel B: non-dominant upper-limb) and association between MBboard-1RM and climbing ability (Panel $C$ : dominant upper-limb, Panel D: non-dominant upper-limb). Data are presented as mean $\pm S D$. * Significantly higher than the control group $(p<0.05)$. 


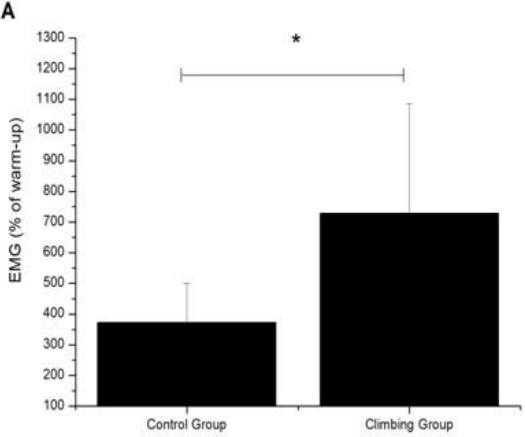

B

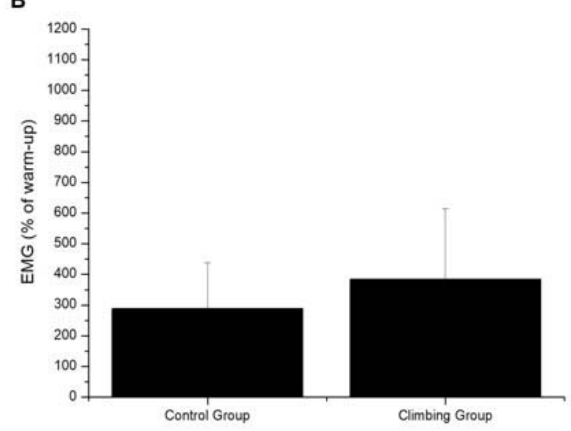

Figure 4

Surface electromyographic activity for the flexor digitorum superficialis (panel A) and posterior deltoid (panel B) during MBboard-1RM. Data are presented as mean \pm SD. EMG: surface electromyography. ${ }^{*}$ Significantly higher than the control group $(p<0.05)$.

\section{Discussion}

The purpose of this study was to develop a low-cost and easy-to-build tool designed to evaluate maximal upper-limb strength in rock climbers in a specific way. Our main results revealed that: i) MBboard-1RM was able to discriminate between individuals with different climbing experience levels, ii) MBboard1RM was significantly correlated with climbing ability self-reported by rock climbers, and iii) good reliability of MBboard-1RM was found when analysed within trials. Taken together, these findings suggest that the MBboard is a valid and reliable tool to assess rock climbingspecific upper-limb strength.

Previous studies have widely recommended the development of new tools for rock climbing-specific performance measurements (Giles et al., 2006; Sheel et al., 2004; Watts, 2004). To the best of our knowledge, this is the first study to develop a low-cost and easy-to-build tool (i.e., MBboard) to assist in the evaluation of upper-limb strength in rock climbers. The MBboard was designed in order to meet the specific upper body muscular demand of rock climbing which has been characterized by high-intensity isometric contraction of the forearms combined with dynamic whole-body movements (Limmer et al., 2019). Considering that there was no method universally accepted as gold standard for the measurement of maximal strength in rock climbers (Michailov et al., 2018), previous studies have adopted the strategy to obtain construct validity evidence of field and laboratory tests by comparison between individuals with different rock climbing experience and/or by association with climbing ability self-reported by rock climbers (Brent et al., 2009; Grant et al., 1996; López-Rivera and González-Badillo, 2019; Michailov et al., 2018). In the current study, the RCG presented superior MBboard-1RM performance when compared with the CG for both the dominant $(p<0.01)$ and the non-dominant $(p<0.01)$ upper-limb. Our results also revealed that MBboard-1RM was 
positively correlated with climbing ability, indicating that better rock climbers were able to produce higher values of MBboard-1RM. Collectively, these findings denote the construct validity evidence of the MBboard for the measurement of maximal upper-limb strength in rock climbers in a specific way.

Another interesting result of the present study was that EMG activity of the flexor digitorum superficialis had a similar response to MBboard-1RM performance, with the RCG showing higher values than the CG (Figure 3, panel A). In contrast, there was no significant difference between groups for EMG activity of the posterior deltoid (Figure 3, panel B). This is in accordance with previous results showing the importance of neuromuscular variables, such as finger flexors EMG activity, for rock climbing performance (Cutts and Bollen, 1993; Limonta et al., 2008; Magiera et al., 2013; Quaine et al., 2003; Vigouroux and Quaine, 2006). Vigouroux et al. (2015), for example, reported a significantly higher finger flexor muscles activation of rock climbers when compared with non-climbers. Deyhle et al. (2015) found a great reduction in digit flexion EMG activation after a fatiguing climbing task, suggesting that finger flexor muscle groups were one of the most recruited muscles for climbing on an overhanging wall. Taken together, these findings suggest that ability of the MBboard to assist in the assessment of rock climbing-specific strength may be related in part to the finger flexor muscles activation, probably reflecting the specific adaptations resulting from long-term practice of this sports discipline.

From the practical perspective, it is also important to determine the reliability before using a new performance test to accurately monitor and prescribe training protocols. A reliable test is one that has small changes in mean values, a small within-individual variation, and a high test-retest correlation (Stöggl et al., 2006). The reliability of performance tests can be statistically determined by different ways, with the ICC being considered one of the most appropriate statistical analysis for this proposal (Impellizzeri and Marcora, 2009). Although none of the rock climbers had used the MBboard before, our findings revealed good reliability of MBboard-1RM for both dominant (ICC $=0.79, p=$ 0.01 ) and non-dominant (ICC $=0.85, p<0.01$ ) upper-limbs. In the present study, we adopted only one familiarization trial in the experimental design before the experimental trials, which appears to be sufficient to mitigate the practice effects on MBboard-1RM performance. Therefore, this suggests that, using at least one familiarization session, it is possible to achieve good reliability of MBboard-1RM for detecting rock climbing-specific strength.

Some limitations of the present study should be recognized. First, when climbing a route or during a bouldering task, climbers have to use a wide variety of artificial handholds with different shapes and size to reach the top. This may impose different hand and finger positions to achieve optimal grip strength. Second, we were able to analyse only the unilateral seated cable row exercise, while climbing routes are typically built on artificial walls with different inclinations, which may require a wide variety of movements. In this respect, further studies are required to investigate the influence of other artificial handholds, finger positions, and upper body movements on the validity and reliability of the MBboard for detecting rock climbing-specific strength.

In conclusion, the findings of the current study provided construct evidence of validity and reliability of the MBboard for the measurement of rock climbing-specific strength. From the practical point of view, these findings open a new path for the use of the MBboard as a specific auxiliary tool for evaluation and prescribing strength based-training.

\section{Acknowledgements}

The authors thank all participants who took part in this study. We also thank the route setter Andre Berezoski for his contributions to the development of the MBboard, and Ivan Kisil Miskalo for the figures.

Thomas Kisil Marino was supported by a scholarship (USP PUB 2018). 


\section{References}

Baláš J, Michailov M, Giles D, Kodejška J, Panáčková M, Fryer S. Active recovery of the finger flexors enhances intermittent handgrip performance in rock climbers. Eur J Sport Sci, 2015; 16(7): 764-72

Bertuzzi RC, Franchini E, Kokunbun E, Kiss MA. Energy system contributions in indoor rock climbing. Eur J Appl Physiol, 2007; 101: 293-300.

Bourdin C, Teasdale N, Nougier V. Attentional demands and the organization of reaching movements in rock climbing. Res Q Exercise Sport, 1998; 69(4): 406-10

Brent S, Draper N, Hodgson C, Blackwell G. Development of a performance assessment tool for rock climbers. Eur J Sport Sci, 2009; 9(3): 159-167

Brown LE, Weir JP. Procedures recommendation I: accurate assessment of muscular strength and power. J Exerc Physiol, 2001; 4: 1-21

Coley B, Jolles BM, Farron A, Pichonnaz C, Bassin JP, Aminian K. Estimating dominant upper-limb segments during daily activity. Gait Posture, 2008; 27: 368-375

Coppieters M, Stappaerts K, Janssens K, Jull G. Reliability of detecting "onset of pain" and "submaximal pain" during neural provocation testing of the upper quadrant. Physiother Res Int, 2002; 7(3): 146-156

Cutts A, Bollen SR. Grip strength and endurance in rock climbers. Proc Inst Mech Eng H, 1993; 207(2): 87-92

Damasceno MV, Duarte M, Pasqua LA, Lima-Silva AE, MacIntosh BR, Bertuzzi R. Static stretching alters neuromuscular function and pacing strategy, but not performance during a 3-km running time-trial. PLoS One, 2014; 9(6)

Damasceno MV, Lima-Silva AE, Pasqua LA, Tricoli V, Duarte M, Bishop DJ, Bertuzzi R. Effects of resistance training on neuromuscular characteristics and pacing during 10-km running time trial. Eur J Appl Physiol, 2015; 115: 1513-1522

Deyhle MR, Hsu H-S, Fairfield TJ, Cadez Schmidt TL, Gurney BA, Mermier CM. Relative Importance of Four Muscle Groups for Indoor Rock Climbing Performance. J Strength Cond Res, 2015; 29(7): 2006-2014

Draper N, Giles D, Schöffl V, Fuss FK, Watts P, Wolf P, Baláš J, Espana-Romero V, Gonzalez GB, Fryer S, Fanchini M, Vigouroux L, Seifert L, Donath L, Spoerri M, Bonetti K, Phillips K, Stöcker U, Bourassa-Moreau F, Garrido I, Drum S, Beekmeyer S, Ziltener JL, Taylor N, Beeretz I, Mally F, Amca AM, Linhart C, Abreu E. Comparative grading scales, statistical analyses, climber descriptors and ability grouping: International Rock Climbing Research Association position statement. Sports Technology, 2015; 8(3-4): 88-94

Giles LV, Rhodes EC, Taunton JE. The physiology of rock climbing. Sports Med, 2006; 36(6): 529-545

Grant S, Hynes V, Whittaker A and Aitchison T. Anthropometric, strength, endurance and flexibility characteristics of elite and recreational climbers. J Sports Sci, 1996; 14: 301-309

Grant S, Hasler T, Davies C, Aitchison TC, Wilson J, Whittaker A. A comparison of the anthropometric, strength, endurance, and flexibility characteristics of female elite and recreational climbers and non-climbers. J Sports Sci, 2001; 19: 499-505

Impellizzeri FM, Marcora SM. Test validation in sport physiology: lessons learned from clinimetrics. Int J Sports Physiol Perform, 2009; 4(2): 269-277

Koukoubis TD, Cooper LW, Glisson RR, Seaber AV, Feagin JA. An electromyographic study of arm muscles during climbing. Knee Surg Sport Tr, 1995; 3: 121-124

Limmer M, Buck S, Marées M, Roth R. Acute effects of kinesio taping on muscular strength and endurance parameters of the finger flexors in sport climbing: A randomised, controlled crossover trial. Eur J Sport Sci, 2019; 1-10

Limonta E, Cè E, Veicsteinas A, Esposito F. Force control during fatiguing contractions in elite rock climbers. Sport Sci Health, 2008; 4(3): 37-42

López-Rivera E, González-Badillo JJ. Comparison of the Effects of Three Hangboard Strength and Endurance Training Programs on Grip Endurance in Sport Climbers. J Hum Kinet, 2019; 66: 183-195

Magiera A, Roczniok R, Maszczyk A, Czuba M, Kantyka J, Kurek P. The structure of performance of a sport climber. J Hum Kinet, 2013; 28(36): 107-117

Magiera A, Roczniok R, Sadowska-Krepa E, Kempa K, Placek O, Mostowik A. The effect of physical and mental stress on the heart rate, cortisol and lactate concentrations in rock climbers. J Hum Kinet, 2018; 31(65): 111-123 
Mermier CM, Janot JM, Parker DL, Swan JG. Physiological and anthropometric determinants of sport climbing performance. Br J Sports Med, 2000; 34(5): 359-365

Michailov ML, Baláš J, Tanev SK, Andonov HS, Kodejška J, Brown L. Reliability and Validity of Finger Strength and Endurance Measurements in Rock Climbing. Res Q Exercise Sport, 2018; 1-9

Michailov M. Workload characteristics, performance limiting factors and methods for strength and endurance training in rock climbing. Med Sportiva, 2014; 18(3): 97-106

Quaine F, Martin L, Blanchi JP. Effect of a leg movement on the organization of the forces at the holds in a climbing position 3-D kinetic analysis. Hum Mov Sci, 1997; 16(2-3): 337-346

Quaine F, Vigouroux L, Martin L. Finger Flexors Fatigue in Trained Rock Climbers and Untrained Sedentary Subjects. Int J Sports Med, 2003; 24: 424-427

Sheel AW, Seddon N, Knight A, McKenzie DC, Warburton DE. Physiological responses to indoor rockclimbing and their relationship to maximal cycle ergometry. Med Sci Sports Exerc, 2003; 35(7): 1225- 1231

Stöggl T, Lindinger S, Müller E. Reliability and validity of test concepts for the crosscountry skiing sprint. Med Sci Sports Exerc, 2006; 38: 586-591

Thompson EB, Farrow L, Hunt JEA, Lewis MP, Ferguson RA. Brachial artery characteristics and microvascular filtration capacity in rock climbers. Eur J Sport Sci, 2014; 1-9

Vigouroux L, Quaine F. Fingertip force and electromyography of finger flexor muscles during a prolonged intermittent exercise in elite climbers and sedentary individuals. J Sport Sci, 2006; 24(2): 181-186

Vigouroux L, Goislard de Monsabert B, Berton E. Estimation of hand and wrist muscle capacities in rock-climbers. Eur J Appl Physiol, 2015; 115: 947-957

Watts PB, Martin DT, Durtschi S. Anthropometric profiles of elite male and female competitive sport rock climbers. J Sport Sci, 1993; 11(2): 113-117

Watts PB. Physiology of difficult rock climbing. Eur J Appl Physiol, 2004; 91(4): 361-372

\section{Corresponding author:}

\section{Romulo Bertuzzi}

School of Physical Education and Sport - University of São Paulo

Address: Av. Prof. Melo Moraes, 65 - Cidade Universitária

São Paulo - SP. Brazil.

ZIP code: 05508-030

Phone Number: +55 (11) 97454-7480

Email: bertuzzi@usp.br 